\title{
Physical Therapy Approaches in the Treatment of Low Back Pain
}

Edward A. Shipton

Received: July 16, 2018 / Published online: September 18, 2018

(C) The Author(s) 2018

\section{ABSTRACT}

Globally, in 2016, low back pain (LBP) contributed 57.6 million of total years lived with disability. Low Back Pain Guidelines regularly recommend the use of physical exercise for non-specific LBP. Early non-pharmacological treatment is endorsed. This includes education and self-management, and the recommencement of normal activities and exercise, with the addition of psychological programs in those whose symptoms persist. The aim of physical treatments is to improve function and prevent disability from getting worse. There is no evidence available to show that one type of exercise is superior to another, and participation can be in a group or in an individual exercise program. Active strategies such as exercise are related to decreased disability. Passive methods (rest, medications) are associated with worsening disability, and are not recommended. The Danish, United States of America, and the United Kingdom Guidelines recommend the use of exercise on its own, or in combination with other non-pharmacological therapies. These

Enhanced digital features To view enhanced digital features for this article go to https://doi.org/10.6084/ m9.figshare.7039769.

\section{E. A. Shipton ( $\square)$}

Department of Anaesthesia, University of Otago, Christchurch, New Zealand

e-mail: shipton@shipton.nz include tai chi, yoga, massage, and spinal manipulation. Public health programs should educate the public on the prevention of low back pain. In chronic low back pain, the physical therapy exercise approach remains a firstline treatment, and should routinely be used.

Keywords: Disability prevention; Improved function; Low back pain; Physical therapy

\section{INTRODUCTION}

Low back pain (LBP) has become an increasing problem around the world [1]. It is increasing as a result of an ageing and expanding world population [1]. The years lived with disability from low back pain have gone up by more than $50 \%$ since 1990 , particularly in low-income and middle-income countries $[1,2]$. In general, it is related to smoking, obesity, sedentary occupations, and to low socioeconomic status (with poor quality of life and limited resources) [2]. In low-income and middle-income countries, disability and costs from low back pain will rise in the future, especially where health systems are delicate and cannot cope with this increasing burden [2]. Globally, in 2016, low back pain contributed 57.6 million $[95 \%$ uncertainty interval (UI) 40.8-75.9 million (7.2\%, 6.0-8.3)] of total years lived with disability (YLDs) [3]. 
The cultural, social, and political environment of back pain can influence the perception of pain, the disability created, and the use of health care [4]. High-quality economic appraisals of looking at surgery when compared to conservative care (with the use of different treatment options) are needed in chronic low back pain (CLBP) patients [5]. This article is based on previously conducted studies and does not contain any studies with human participants or animals performed by the author.

\section{Low Back Pain Clinical Guidelines}

Guidelines recommend the non-pharmacological and non-invasive management [6]. These include the provision of advice to stay active and the use of patient education and exercise therapy [6]. Guidelines regularly recommend the use of physical exercise for non-specific LBP [7]. Guidelines endorse the cautious use of imaging, of medication, and of surgery [8]. A risk stratification tool is recommended in the National Institute for Health and Care Excellence (NICE) guidelines [7], so that treatments can be co-ordinated to each risk subgroup [8].

Patients with low back pain can be triaged using a clinical assessment [9]. This should include history-taking, physical examination, and neurological tests to recognize radicular features [10]. With low back pain, patients should be screened for 'red flags' to exclude serious pathologies, and diagnostic tests (such as imaging) carried out if suspected [11].

Psychosocial risk factors (yellow flags using prognostic screening tools) should be assessed to predict poorer outcomes $[10,11]$. There can be mutual decisions made with the patient as to whether simpler and less-intensive management is called for. If there is no improvement after 4 weeks, and a serious pathology or radiculopathy is suspected, then specialist consultation is recommended [10].

Examples of simpler management include guidance and reassurance on self-management, guidance to stay active and avoid bed rest, guidance to return to normal activities, or referral for a group or an individual exercise program [7]. This could be combined with manual or psychological therapies in a combined rehabilitation programme [7].

\section{PREVENTION}

Public health programs that challenge obesity and low physical activity levels should be developed and provide the forum for decreasing the effects of low back pain on daily living [12]. In CLBP, evidence for prevention and treatment often comes from high-income countries. Whether or not these guideline recommendations are applicable for low-income and middleincome countries, remains unknown [8]. Public health programs and their urgency will differ in high-income countries when compared to lowincome and middle-income countries as well [8]. An obstacle in altering health pathways concerns the existing models of health-care reimbursement [8]. It is useful to have the whole health pathway for low back pain mapped out, from the first contact all the way through to specialized care [8].

Health-care professionals should deliver regular education concerning the causes, the mechanisms, the natural history, and prognosis of low back pain, and promote the benefits of physical activity and exercise [12].

Exercise alone or in combination with education has shown moderate-quality evidence that this is effective for prevention of LBP [13]. Its preventive effect was found to be high, with a pooled relative risk of 0.55 (95\% CI 0.41-0.74) [13]. With intensive programs, exercise then can be focused on secondary prevention.

In 2014, a systematic review and meta-analysis found only four pediatric trials in pediatric low back pain [14]. This casts doubt regarding the evidence for treatment of back pain in children [14]. There was moderate-quality evidence that education was not effective in children [14]. There was very low quality evidence that ergonomically designed furniture prevented low back pain [14].

A recent meta-analysis was performed on the prevention of low back pain using exercise [15]. Exercise on its own was able to decrease the risk of LBP by $33 \%$ (risk ratio $=0.67 ; 95 \%$ CI 0.53 , $0.85, I^{2}=23 \%$, where $I^{2}$ describes the percentage 
of variation across studies that is due to heterogeneity rather than chance; eight randomized controlled trials; $n=1634$ ) [15]. When exercise was combined with education, it reduced the risk by $27 \%$ (risk ratio $=0.73 ; 95 \%$ CI $0.59,0.91, I^{2}=6 \%$; six trials; $n=1381$ ) [15]. The intensity of LBP and the accompanying disability from LBP were also decreased in the exercise groups when compared to the control groups [15]. The analysis concluded that exercise diminished the risk of LBP and its associated disability [15]. A mixture of strengthening with either stretching or aerobic exercises when performed two to three times per week could sensibly be endorsed for the prevention of LBP in the general population [15].

\section{Management of Acute Low Back Pain}

For acute non-specific low back pain that does not have serious pathology (red flags have been excluded), initial reassurance, advice to stay active and self-management are all that is needed $[6,7,9,11]$. Self-management can include self-exercises and education from reading booklets or being involved in on-line education for low back pain [16] (Table 1).

Table 1 Management of acute low back pain (without serious pathology)

Acute low back pain (without serious pathology)
Initial reassurance, guidance to stay active and avoid bed
rest, and provide guidance on self-management
Self-management can include self-exercises and
education from reading booklets or being involved in
online education for low back pain
Primary conservative physical treatment may include
exercises, superficial heat, and manual therapy
Guidance to return to normal activities, or referral for
an individual or group exercise program
Pharmacological therapies include nonsteroidal anti-
inflammatory drugs (NSAIDs) and weak opioids for
brief periods (paracetamol is not recommended)
Progress should be reviewed in $7-14$ days

Recommended primary conservative physical treatment preferences include manual therapy, exercise, and superficial heat [17]. There is low evidence that low-level laser therapy is more effective than sham laser for pain $[18,19]$. Limited evidence shows that acupuncture is modestly effective for acute low back pain [18]. The McKenzie method of mechanical diagnosis and therapy (MDT) is designed to categorize patients into homogeneous subgroups (derangement, dysfunction, or postural syndrome) [20]. This is in order to direct treatment with specific exercises and postural advice [20]. In acute LBP, moderate- to high-quality evidence exists that MDT is not superior to other rehabilitation interventions in decreasing pain and disability [20]. Back schools use varying exercises and educational methods. There is very low-quality evidence that back school is more effective than no treatment (mean difference $(\mathrm{MD})-6.10,95 \%$ confidence interval $(\mathrm{CI})-10.18$ to -2.01 ) in acute low back pain [21].

In acute low back pain, when pharmacological therapies are considered, these would include nonsteroidal anti-inflammatory drugs (NSAIDs), skeletal muscle relaxants, and weak opioids for brief periods (paracetamol is not recommended) $[9,10]$.

For acute low back pain, most patients improve with or without therapy [18]. The magnitude of pain benefits is small to moderate and generally short term [18]. Progress should be reviewed in 7-14 days [7]. Guidance should be given to return to normal activities [8], or referral made for an individual or group exercise program [7].

There has been no recommendation as to the level of pain allowed during exercise, and to the level of pain tolerated at each stage of the exercise progression [6]. A systematic review protocol has recently been published in order to study the effect of using a differentiation of exercises based on the amount of low back pain experienced by patients in primary care [6].

In summary, guidelines recommend early non-pharmacological treatment that includes education and self-management, and the recommencement of normal activities and 
exercise, with the addition of psychological programs in those whose symptoms persist [8].

\section{PHYSICAL TREATMENT PREFERENCES}

In low back pain, guidelines (as stated above) promote the avoidance of bed rest, and the continuation with activities as usual [22]. The aim of physical treatments is to improve function, and to prevent disability from getting worse [8]. In chronic low back pain, exercise therapy has become a first-line treatment and should be routinely used [8].

Should recovery be slow in patients with risk factors for developing persistent disabling pain, early supervised exercise therapy can be considered [23]. If low back pain persists for more than 12 weeks, physical treatments that encompass a graded activity or exercise programs that focus on improvements in function, are recommended [8]. In fact, in low back pain greater than 12 weeks, exercise is a first-line treatment that should be considered for routine use [8]. All recent clinical practice guidelines endorse exercise therapy in persistent low back pain [10]. Yet access to structured exercise programs remains erratic [8].

In clinical practice guidelines, there remain large inconsistencies in the type of exercise program (yoga, stretching, hydrotherapy exercises, tai chi, McKenzie exercise approach and back schools) needed, and in the way that it is delivered (group exercise individual programs, or supervised home exercise) [10]. Choice may ultimately depend on patients' preferences and on the experience of the treating therapist [10]. Clinical practice guidelines now suggest that a diversity of types of exercises should be used [10]. Exercise induces pain relief by the activation of central inhibitory paths [18]. Mechanisms involving opioids, serotonin, and $N$ methyl-D-aspartate (NMDA) in the rostral ventromedial medulla stimulates pain relief associated with exercise [24].

Back pain prevalence is low in children, but increases during adolescence [9]. A systematic review has found that prevention and treatment interventions having an exercise component are most likely to be effective [14].

There is no evidence available to show that one type of exercise is superior to another [8]. In deciding on the type of exercise to be used, guidelines should, however, incorporate individual preferences, needs, and capabilities [8]. Movement control tests, laterality judgment, and two-point discrimination show the highest level of known-groups validity for people with chronic low back pain [25]. Nonetheless, the reliability of these measurement tools has yet to be established.

In chronic low back without serious pathology, recommended primary conservative physical treatment preferences include exercise, yoga, biofeedback, progressive relaxation, massage, manual therapy, and interdisciplinary rehabilitation [17] (Table 2).

In spinal pain with radiculopathy, exercise and spinal manipulation can be used [17]. However, some guidelines do not endorse the use of passive therapies, or make them optional in patients unresponsive to other treatments [26]. These include massage, spinal manipulation or mobilization, and acupuncture [26].

Other passive physical or electrical methods, such as short-wave diathermy, interferential therapy transcutaneous electrical nerve stimulation (TENS), back supports, traction, and ultrasound have been largely found to be ineffective, and are not recommended [22, 27, 28]. A systematic review has shown that high-quality randomized controlled trials (RCTs) to determine the effects of TENS are needed due to the low quality of present studies, wherein adequate parameters and timing of assessment were not consistently reported or used [29].

\section{ASSOCIATION WITH PSYCHOSOCIAL FACTORS}

In the treatment of back pain by physiotherapists, an association exists with psychosocial factors, such as self-efficacy, catastrophizing, fear of movement, and pain and disability outcomes [30]. A recent systematic review looked at the psychosocial factors related to change in pain and disability outcomes in chronic low 
Table 2 Management of chronic low back pain (without serious pathology)

\section{Chronic low back pain (without serious pathology)}

Triage using a clinical assessment (history-taking, physical examination, and neurological tests (to recognize radicular features)

Patients should be screened for 'red flags' to exclude serious pathologies, and diagnostic tests (such as imaging) only carried out if suspected

Patients should be screened for psychosocial risk factors ('yellow flags' such as low self-efficacy, catastrophizing, fear of movement) to predict poorer outcomes

Use a risk stratification tool (such as STarT)

Non-pharmacological and non-invasive management treatment is recommended that includes education and self-management, and the recommencement of normal activities and exercise, with the addition of psychological programs in those whose symptoms persist (multidisciplinary treatments)

Primary conservative physical treatment exercises include walking, Pilates, tai chi, yoga, progressive relaxation (and massage, and manual therapy in some guidelines)

No evidence available to show that one type of exercise is superior to another

Choice may ultimately depend on patients' preferences and on the experience of the treating therapist

A diversity of types of exercises should be used

Physical therapy exercise approach remains a first-line treatment, and should routinely be used

Referral could be for an individual or group exercise program

Passive physical therapies (massage, spinal mobilization, acupuncture, and spinal manipulation with radiculopathy) are not usually endorsed, or are optional in some guidelines

Passive methods (rest, medications) are associated with worsening disability, and are not recommended
Table 2 continued

Pharmacological therapies if used include nonsteroidal anti-inflammatory drugs (NSAIDs) and antidepressants at the lowest effective dose and for the least possible time

Injections, denervation procedures, and the use of surgery are generally not endorsed

No improvement after 4 weeks, or pathology or radiculopathy suspected, then specialist consultation

back pain patients that were treated by physiotherapists [30]. An association was found between psychosocial factors, such as self-efficacy, catastrophizing, and fear of movement, and pain and disability outcomes [30].

\section{PILATES}

An exercise system centering on controlled movement, breathing, and stretching is known as Pilates [31]. A systematic literature review (using the Preferred Reporting Items for Systematic Reviews and Meta-Analyses or PRISMA guidelines) has been published. It included 23 studies (published between 2005 and 2016) [31]. Most clinical trials in the past 5 years have found Pilates to be an effective rehabilitation tool that has resulted in desired outcomes, such as reducing pain and disability [31].

\section{YOGA}

A Cochrane systematic review demonstrated a slight functional improvement when yoga is used for chronic non-specific low back pain with a slight reduction in pain; it heightened the chance of clinical improvement [32]. In some people, however, it was found to increase their back pain [32].

\section{WALKING}

The advantage of walking is that it is easy to carry out. In chronic low back pain, a meta- 
analysis of nine suitable randomized controlled trials was performed to understand the effectiveness of walking on disability, pain, and quality of life at post intervention and at followup visits [33]. The duration of follow-up, namely: short-term ( $<3$ months), intermediateterm (between 3 and 12 months), and longterm (>12 months), was used to analyze the data [33]. In short- and intermediate-term follow-ups, walking was found (with low- to moderate-quality evidence) to be as effective as other non-pharmacological interventions in decreasing disability and pain, and was recommended [33].

\section{MOBILIZATION AND MANIPULATION THERAPIES}

A recent systematic literature review and metaanalysis has examined mobilization and manipulation for treating chronic low back pain [34]. Bias was assessed using the Scottish Intercollegiate Guidelines Network criteria. The confidence in effect estimates was defined using the Grading of Recommendations, Assessment, Development, and Evaluation (GRADE) system. Nine trials (1176 patients) provided sufficient data. Following treatment, the standardized mean difference for a decrease in pain was standardized mean difference $(\mathrm{SMD})=-0.28$, [95\% confidence interval (CI) -0.47 to -0.09 , $p=0.004 ; I^{2}=57 \%$ (where $I^{2}$ describes the percentage of variation across studies that is due to heterogeneity rather than chance)] [34]. When mobilization or manipulation was compared to other active therapies, seven trials (923 patients) showed the decrease in disability to be a $\mathrm{SMD}=-0.33, \quad[95 \%$ CI -0.63 to -0.03 , $\left.p=0.03 ; I^{2}=78 \%\right]$ [34].

Subgroup analyses showed that mobilization when compared to other active comparators (that included exercise), significantly decreased pain $(\mathrm{SMD}=-0.20$, [95\% CI -0.35 to -0.04 ; $\left.p=0.01 ; I^{2}=0 \%\right]$ ), but not disability (SMD = $-0.10,[95 \%$ CI -0.28 to $0.07 ; p=0.25$; $\left.I^{2}=21 \%\right]$ ) [34].

Subgroup analyses showed that manipulation when compared to other active comparators (that included physical therapy and exercise), significantly decreased pain and disability (SMD $=-0.43$, [95\% CI -0.86 to 0.00 ; $p=0.05, \quad I^{2}=79 \% ; \quad \mathrm{SMD}=-0.86, \quad 95 \% \quad \mathrm{CI}$ -1.27 to $\left.-0.45 ; \quad p<0.0001, \quad I^{2}=46 \%\right]$, respectively) [34].

A systematic review and meta-analysis of manipulation and mobilization in the treatment of chronic low back pain published in 2018, found moderate-quality evidence that manipulation and mobilization decreased pain and increased function [34]. Manipulation seemed to be more effective than mobilization [34], although both were safe [34].

\section{MOVEMENT CONTROL EXERCISES}

A systematic review and meta-analysis of the effectiveness of movement control exercise on patients with non-specific low back pain and movement control impairment (MVCE), was recently carried out [35]. There was 'very low to moderate quality evidence of a positive effect of MVCE on disability, both at the end of treatment (SMD - 0.38; 95\% CI - 0.68, - 0.09), and after 12 months (SMD 0.37; 95\% CI - 0.61, - 0.04) [35]. Pain intensity became significantly decreased after MVCE at the end of treatment (SMD - 0.39; 95\% CI - 0.69, - 0.04), but not after 12 months (SMD - 0.27; 95\% CI - 0.62, 0.09) [35].

\section{TECHNOLOGY-SUPPORTED EXERCISE THERAPY}

Technological systems, such as electromyography feedback (EMG-FB) provide technologysupported exercise therapy (TSET), and have been progressed to benefit exercise therapy for low back pain [36]. In patients with low back pain, a recent systematic review found that TSET improved pain, disability, and quality of life [36]. However, for most technologies, only a limited number of RCTs were available, and solid conclusions regarding the effectiveness of individual technological systems could not be made [36]. 


\section{MCKENZIE METHOD OF MECHANICAL DIAGNOSIS AND THERAPY (MDT)}

Using the information obtained from the McKenzie Method of Mechanical Diagnosis and Therapy (MDT) assessment, the clinician will then prescribe specific exercises and advice regarding postures to adopt and postures to temporarily avoid [20]. A recent literature review with meta-analysis in patients with chronic LBP found moderate- to high-quality evidence that MDT was superior to other rehabilitation interventions in reducing pain and disability, but was dependent on the type of intervention used for comparison to MDT [20].

\section{PREGNANCY}

Another meta-analysis found that even during and after pregnancy, osteopathic manipulative treatment for low back and pelvic girdle pain during and after pregnancy gave clinically relevant benefits [37]. A meta-analysis of randomized controlled trials (RCTs) published in 2018 showed that exercise decreased the risk of low back pain in pregnancy by $9 \%$ [pooled risk ratio $(\mathrm{RR})=0.91 ; 95 \%$ CI 0.83-0.99; $I^{2}=0 \%$, seven trials; $n=1175$ ] [38]. However, there was no protective effect on pelvic girdle pain $\left(\mathrm{RR}=0.99 ; \quad \mathrm{CI} \quad 0.81-1.21 ; I^{2}=0 \%\right.$; four RCTs; $n=565)$, or on lumbarpelvic pain $\left(\mathrm{RR}=0.96 ;\right.$ CI $0.90-1.02 ; I^{2}=0 \%$; eight RCTs; $n=1737$ ) [38]. In lumbar-pelvic pain, exercise was able to prevent new episodes of sick leave $\left(\mathrm{RR}=0.79\right.$; CI $0.64-0.99 ; I^{2}=0 \%$; three RCTs; $n=1168$ ) [38].

\section{BACK SCHOOLS}

A recent Cochrane systematic review of RCTs evaluating the effectiveness of back schools was undertaken [21]. Low-quality evidence showed back schools to be no better than exercise in the intermediate-term (mean difference or MD -4.46 ; $95 \% \mathrm{CI}-19.44$ to 10.52 ), as well as at long-term follow-up (MD 4.58; 95\% CI - 0.20 to 9.36) [21]. It remains unclear whether or not back schools are effective for chronic low back pain. At the long-term follow-up, very lowquality evidence showed passive physiotherapy to be better than back school (MD 9.60; 95\% CI 3.65-15.54) [21].

\section{OUTCOME MEASURES}

Consensus procedures and systematic reviews on existing patient-reported outcome measures (PROMs) show the Roland Morris Disability Questionnaire-24 item (RMDQ-24) and the Oswestry Disability Index 2.1a (ODI 2.1a) to be the instruments that have most often been used to measure physical functioning [39]. The third most frequently used PROM for physical functioning was the Quebec Back Pain Disability Scale (QBPDS) [40].

A systematic review has recently been undertaken to investigate the reliability of physical functioning tests in patients with low back pain, and to investigate their reliability [41]. The following tests recorded good overall test-retest reliability, namely: the flexor endurance test $(\mathrm{ICC}=0.90-0.97)$; the extensor endurance test [intra-class correlation coefficient $($ ICC $)=0.93-0.97)$ ]; the 50-foot walking test (ICC $=0.76-0.96$ ); the 5-min walking test (ICC $=0.89-0.99) ; \quad$ the sit-to-stand test (ICC $=0.91-0.99)$; the loaded forward reach test (ICC $=0.74-0.98)$, and; the shuttle walk test (ICC $=0.92-0.99) \quad[41]$. Only the Biering-Sörensen test (ICC $=0.88-0.99$ ) was found to have an overall good inter-rater reliability. None of the clinical tests used had good intra-rater reliability [41].

\section{CLINICAL GUIDELINES}

Clinical guidelines are used to encourage dependable best practice, to decrease unnecessary discrepancy, and eliminate low-value interventions [7]. In low back pain, little evidence is available to guide health care professionals on how to advance the use and uptake of recommended evidence-based practice [42].

Self-management can include self-exercises and education from reading booklets or being 
involved in on-line education, or making use of unevaluated smartphone applications (apps) for low back pain [43]. Some guidelines recommend self-management in treating chronic low back pain, but strong evidence of its effectiveness is lacking; this needs to be improved [44]. For non-specific LBP, most guidelines recommend physical exercise [7].

The STarT Back Screening Tool is a validated nine-item patient self-report questionnaire that categorizes LBP patients as being at a low, medium, or high-risk of developing persistent non-specific LBP [45]. The NICE guideline recommends using a risk stratification tool (such as STarT or Orebro) to inform shared decisionmaking about whether or not a patient can be managed with simpler and less-intensive support [7].

Examples of this are to reassure, give advice on keeping active, and provide guidance on selfmanagement or referral. Referral could be for a range of possible rehabilitation options. These include a group or individual exercise program (with or without manual or psychological therapies), or to a 'combined physical and psychological' rehabilitation program [7]. According to the NICE guidelines, combined psychological and physical rehabilitation treatments are recommended where prior treatments have been ineffective, and where substantial psychological obstacles to recovery exist [7].

For chronic LBP, both Canadian and United States of America (USA) guidelines favor multidisciplinary pain management programmes [7]. The Danish [27], United States of America (USA) [28], and the United Kingdom (UK) [22] guidelines recommend the use of exercise on its own, or in combination with other non-pharmacological therapies. These include tai chi (USA), and yoga (USA), massage (USA and UK), and spinal manipulation (Danish, USA, and UK).

\section{CURRENT PRACTICE}

Based on the United States, Belgian, Danish, and United Kingdom guidelines, a recent review has summarized recommended changes in management [9]. For more chronic non-specific low back pain, non-pharmacological therapies, such as physical (exercise) and psychological (cognitive behavioral therapy), should be tried before pharmacological interventions, such as nonsteroidal anti-inflammatory drugs (NSAIDs) and antidepressants, are considered $[9,10]$. Multidisciplinary treatments are recommended as well [9]. All pharmacological interventions should be at the lowest effective dose and for the least possible time [9]. Injections, denervation procedures, and the use of surgery are not endorsed [9].

\section{FUTURE STRATEGIES}

Public health programs should educate the public on the prevention of low back pain [8]. Alterations in disability and compensation claims policies may be needed as well [8]. Strategies need to be created and applied to deal with modifiable risk factors for disabling low back pain [12]. Policy-makers both internationally and nationally need to fund and promote the prevention of low back pain [12].

Health care pathways should be developed to ensure that patients are able to consult the right health care professionals for provision of the right treatment at the right time [12]. Improved training of health care professionals could decrease the unnecessary use of medical care [12]. Clinical pathways should be restructured to realize best practice, and interventions to decrease work disability should be incorporated in both health care and occupational environments [8].

Early recognition and the provision of suitable education of low back pain patients at risk for persistence of pain and disability are needed [12]. Passive methods (rest, medications) are associated with worsening disability [12]. Active strategies such as exercise are related to decreased disability [12].

Practice needs to be brought into line with the evidence, and activity and function (plus work participation) should be encouraged [8]. The use of active multidisciplinary rehabilitation should focus on self-management and healthy lifestyles, and assist in a return to work [12]. 
The low back pain area lacks evidence of effective implementation strategies [42]. Implementation trials in the future should assume best-practice implementation research methodology, making use of the Standards for Reporting Implementation Studies of complex interventions guidelines [42].

In patients with low back pain, a metaanalysis of RCTs has been planned to compare physical therapy interventions with placebo or with no intervention [46]. The primary outcomes would be pain intensity and disability [46]. Finally, in chronic low back pain, the physical therapy exercise approach remains a first-line treatment and should be routinely used [8].

\section{ACKNOWLEDGEMENTS}

Funding. No funding or sponsorship was received for this study or the publication of this article.

Authorship. The named author meets the International Committee of Medical Journal Editors (ICMJE) criteria for authorship for this article, takes responsibility for the integrity of the work as a whole, and has given his approval for this version to be published.

Disclosures. Edward A. Shipton has nothing to disclose.

Compliance with Ethics Guidelines. This article is based on previously conducted studies and does not contain any studies with human participants or animals performed by the author.

Data Availability. Data sharing is not applicable to this article as no datasets were generated or analyzed during the current study.

Open Access. This article is distributed under the terms of the Creative Commons Attribution-NonCommercial 4.0 International License (http://creativecommons.org/licenses/ by-nc/4.0/), which permits any noncommercial use, distribution, and reproduction in any medium, provided you give appropriate credit to the original author(s) and the source, provide a link to the Creative Commons license, and indicate if changes were made.

\section{REFERENCES}

1. Clark S, Horton R. Low back pain: a major global challenge. Lancet. 2018;391(10137):2302.

2. Hartvigsen J, Hancock MJ, Kongsted A, Louw Q, Ferreira ML, Genevay S, Hoy D, Karppinen J, Pransky G, Sieper J, Smeets RJ, Underwood M. Lancet low back pain series working group. What low back pain is and why we need to pay attention. Lancet. 2018;391(10137):2356-67.

3. GBD 2016. Disease and Injury Incidence and Prevalence Collaborators. Disease and Injury Incidence and Prevalence Collaborators Global, regional, and national incidence, prevalence, and years lived with disability for 328 diseases and injuries for 195 countries, 1990-2016: a systematic analysis for the Global Burden of Disease Study 2016. Lancet. 2017;390(10100):1211-59.

4. Henschke N, Lorenz E, Pokora R, Michaleff ZA, Quartey JNA, Oliveira VC. Understanding cultural influences in back pain and back pain research. Best Pract Res Clin Rheumatol. 2016;30(6):1037-49.

5. van Dongen JM, Ketheswaran J, Tordrup D, Ostelo RWJG, Bertollini R, van Tulder MW. Health economic evidence gaps and methodological constraints in low back pain and neck pain: results of the Research Agenda for Health Economic Evaluation (RAHEE) project. Best Pract Res Clin Rheumatol. 2016;30(6):981-93.

6. Jorgensen JE, Afzali T, Riis A. Effect of differentiating exercise guidance based on a patient's level of low back pain in primary care: a mixed-methods systematic review protocol. BMJ Open. 2018;8(1):e019742.

7. O'Connell NE, Cook CE, Wand BM, Ward SP. Clinical guidelines for low back pain: a critical review of consensus and inconsistencies across three major guidelines. Best Pract Res Clin Rheumatol. 2016;30(6):968-80.

8. Foster NE, Anema JR, Cherkin D, Chou R, Cohen SP, Gross DP, Ferreira PH, Fritz JM, Koes BW, Peul W, Turner JA, Maher CG, Lancet Low Back Pain Series Working Group. Prevention and treatment of 
low back pain: evidence, challenges, and promising directions. Lancet. 2018;391(10137):2368-83.

9. Almeida M, Saragiotto B, Richards B, Maher CG. Primary care management of non-specific low back pain: key messages from recent clinical guidelines. Med J Aust. 2018;208(6):272-5.

10. Kamper SJ, Yamato TP, Williams CM. The prevalence, risk factors, prognosis and treatment for back pain in children and adolescents: an overview of systematic reviews. Best Pract Res Clin Rheumatol. 2016;30(6):1021-36.

11. Strudwick K, McPhee M, Bell A, Martin-Khan M, Russell T. Best practice management of low back pain in the emergency department (part 1 of the musculoskeletal injuries rapid review series). Emerg Med Australas. 2018;30(1):18-35.

12. Buchbinder R, van Tulder M, Öberg B, Costa LM, Woolf A, Schoene M, Croft P, Lancet Low Back Pain Series Working Group. Low back pain: a call for action. Lancet. 2018;391(10137):2384-8.

13. Steffens D, Maher CG, Pereira LS, et al. Prevention of low back pain: a systematic review and metaanalysis. JAMA Intern Med. 2016;176:199-208.

14. Michaleff ZA, Kamper SJ, Maher CG, Evans R, Broderick C, Henschke N. Low back pain in children and adolescents: a systematic review and meta-analysis evaluating the effectiveness of conservative interventions. Eur Spine J. 2014;23:2046-58.

15. Shiri R, Coggon D, Falah-Hassani K. Exercise for the prevention of low back pain: systematic review and meta-analysis of controlled trials. Am J Epidemiol. 2018;187(5):1093-101.

16. Mork PJ, Bach K. A decision support system to enhance self-management of low back pain: protocol for the selfBACK Project. JMIR Res Protoc. 2018;7(7):e167.

17. Chou R, Côté P, Randhawa K, Torres P, Yu H, Nordin M, Hurwitz EL, Haldeman S, Cedraschi C. The Global Spine Care Initiative: applying evidencebased guidelines on the non-invasive management of back and neck pain to low- and middle-income communities. Eur Spine J. 2018. https://doi.org/10. 1007/s00586-017-5433-8.

18. Chou R, Deyo R, Friedly J, Skelly A, Hashimoto R, Weimer M, Fu R, Dana T, Kraegel P, Griffin J, Grusing S, Brodt ED. Nonpharmacologic therapies for low back pain: a systematic review for an American College of Physicians Clinical Practice Guideline. Ann Intern Med. 2017;166(7):493-505.
19. Basford JR, Sheffield CG, Harmsen WS. Laser therapy: a randomized, controlled trial of the effects of low-intensity Nd:YAG laser irradiation on musculoskeletal back pain. Arch Phys Med Rehabil. 1999;80:647-52.

20. Lam OT, Strenger DM, Chan-Fee M, Pham PT, Preuss RA, Robbins SM. Effectiveness of the McKenzie method of mechanical diagnosis and therapy for treating low back pain: literature review with metaanalysis. J Orthop Sports Phys Ther. 2018;48(6):476-90.

21. Parreira P, Heymans MW, van Tulder MW, Esmail R, Koes BW, Poquet N, Lin CC, Maher CG. Back schools for chronic non-specific low back pain. Cochrane Database Syst Rev. 2017;8:CD011674.

22. UK National Institute for Health and Care Excellence. Low back pain and sciatica in over $16 \mathrm{~s}$ : assessment and management. November 2016. https://www.nice.org.uk/guidance/ng59. Accessed Nov 72017.

23. Chou R, Deyo R, Friedly J, Skelly A, Hashimoto R, Weimer M, Fu R, Dana T, Kraegel P, Griffin J, Grusing S, Brodt E. Noninvasive treatments for low back pain. Rockville: Agency for Healthcare Research and Quality (US); 2016. Report No: 16-EHC004-EF.

24. Da Silva Santos R, Galdino G. Does exercise increase or decrease pain? Central mechanisms underlying these two phenomena. J Physiol Pharmacol. 2018;69(1):3-13.

25. Ehrenbrusthoff K, Ryan CG, Grüneberg C, Martin DJ. A systematic review and meta-analysis of the reliability and validity of sensorimotor measurement instruments in people with chronic low back pain. Musculoskelet Sci Pract. 2018;35:73-83.

26. Wong JJ, Cote P, Sutton DA, et al. Clinical practice guidelines for the noninvasive management of low back pain: a systematic review by the Ontario Protocol for Traffic Injury Management (OPTIMA) Collaboration. Eur J Pain. 2017;21:201-16.

27. Stochkendahl MJ, Kjaer P, Hartvigsen J, et al. National clinical guidelines for non-surgical treatment of patients with recent onset low back pain or lumbar radiculopathy. Eur Spine J. 2018;27:60-75.

28. Qaseem A, Wilt TJ, McLean RM, Forciea MA, Clinical Guidelines Committee of the American College of Physicians. Noninvasive treatments for acute, subacute, and chronic low back pain: a clinical practice guideline from the American College of Physicians. Ann Intern Med. 2017;166:514-30.

29. Resende L, Merriwether E, Rampazo ÉP, Dailey D, Embree J, Deberg J, Liebano RE, Sluka KA. Meta- 
analysis of transcutaneous electrical nerve stimulation for relief of spinal pain. Eur J Pain. 2018;22(4):663-78.

30. Alhowimel A, AlOtaibi M, Radford K, Coulson N. Psychosocial factors associated with change in pain and disability outcomes in chronic low back pain patients treated by physiotherapist: a systematic review. SAGE Open Med. 2018;6:2050312118757387.

31. Byrnes K, Wu PJ, Whillier S. Is Pilates an effective rehabilitation tool? A systematic review. J Bodyw Mov Ther. 2018;22(1):192-202.

32. Wieland LS, Santesso N. A summary of a Cochrane review: yoga treatment for chronic nonspecific low back pain. Eur J Integr Med. 2017;11:39-40.

33. Sitthipornvorakul E, Klinsophon T, Sihawong R, Janwantanakul $P$. The effects of walking intervention in patients with chronic low back pain: a metaanalysis of randomized controlled trials. Musculoskelet Sci Pract. 2018;34:38-46.

34. Coulter ID, Crawford C, Hurwitz EL, Vernon H, Khorsan R, Suttorp Booth M, Herman PM. Manipulation and mobilization for treating chronic low back pain: a systematic review and meta-analysis. Spine J. 2018;18(5):866-79.

35. Luomajoki HA, Bonet Beltran MB, Careddu S, Bauer CM. Effectiveness of movement control exercise on patients with non-specific low back pain and movement control impairment: a systematic review and meta-analysis. Musculoskelet Sci Pract. 2018;36:1-11.

36. Matheve T, Brumagne S, Timmermans AAA. The effectiveness of technology-supported exercise therapy for low back pain: a systematic review. Am J Phys Med Rehabil. 2017;96(5):347-56.

37. Franke H, Franke JD, Belz S, Fryer G. Osteopathic manipulative treatment for low back and pelvic girdle pain during and after pregnancy: a systematic review and meta-analysis. J Bodyw Mov Ther. 2017;21(4):752-62.

38. Shiri R, Coggon D, Falah-Hassani K. Exercise for the prevention of low back and pelvic girdle pain in pregnancy: a meta-analysis of randomized controlled trials. Eur J Pain. 2018;22(1):19-27.

39. Chiarotto A, Terwee CB, Ostelo RW. Choosing the right outcome measurement instruments for patients with low back pain. Best Pract Res Clin Rheumatol. 2016;30(6):1003-20.

40. Chapman JR, Norvell DC, Hermsmeyer JT, et al. Evaluating common outcomes for measuring treatment success for chronic low back pain. Spine. 2011;36:S54e68.

41. Denteneer L, Van Daele U, Truijen S, De Hertogh W, Meirte J, Stassijns G. Reliability of physical functioning tests in patients with low back pain: a systematic review. Spine J. 2018;18(1):190-207.

42. Hodder RK, Wolfenden L, Kamper SJ, Lee H, Williams A, O'Brien KM, Williams CM. Developing implementation science to improve the translation of research to address low back pain: a critical review. Best Pract Res Clin Rheumatol. 2016;30(6):1050-73.

43. Machado GC, Pinheiro MB, Lee $\mathrm{H}$, Ahmed $\mathrm{OH}$, Hendrick P, Williams C, Kamper SJ. Smartphone apps for the self-management of low back pain: a systematic review. Best Pract Res Clin Rheumatol. 2016;30(6):1098-109.

44. Mansell G, Hall A, Toomey E. Behaviour change and self-management interventions in persistent low back pain. Best Pract Res Clin Rheumatol. 2016;30(6):994-1002.

45. Bruyère $\mathrm{O}$, Demoulin $\mathrm{M}$, Brereton $\mathrm{C}$, Humblet $\mathrm{F}$, Flynn D, Hill JC, Maquet D, Van Beveren J, Reginster JY, Crielaard JM, Demoulin C. Translation validation of a new back pain screening questionnaire (the STarT Back Screening Tool) in French. Arch Pub Health. 2012;70(1):12.

46. Almeida MO, Saragiotto BT, Maher CG, Pena Costa LO. Influence of allocation concealment and intention-to-treat analysis on treatment effects of physical therapy interventions in low back pain randomised controlled trials: a protocol of a metaepidemiological study. BMJ Open. 2017;7(9):e017301. 